Ann. Zootech., I973, 22 (I), 55-60.

\title{
RELATION ENTRE L'ACTIVITÉ DES FOLLICULES PILEUX ET LA CROISSANCE CORPORELLE DES BOVINS A VIANDE
}

\author{
M. BROCHART et C. LERICOLLAIS \\ Laboratoire de Physiopathologie de la Nutrition, \\ Centre de Recherches de Clermont-Ferrand, I. N. R. A., \\ Saint Genès Champanelle, \\ 63110 Beaumont
}

\section{RÉSUMÉ}

L'expérience a porté sur 55 taurillons de 4 races, recevant des régimes alimentaires différents. L'activité des follicules pileux a été mesurée de façon indirecte par le taux de racines en anagène des poils, obtenus par épilation. Quatre prélèvements de poils ont été effectués au cours de l'engraissement, en décembre, mars, avril et, pour le dernier prélèvement, d'une part lors de l'abattage, qui a eu lieu de mai à juillet pour 43 animaux de races et de régimes alimentaires variés, d'autre part le Io août, c'est-à-dire de o à 59 jours avant l'abattage, pour I 2 Charolais ayant un même régime.

Les taux de racines de poils en anagène des trois premiers prélèvements étaient très élevés ; on a observé une baisse du taux de racines de poils en anagène pour le dernier prélèvement. On a obtenu des corrélations hautement significatives entre le taux de racines de poils en anagène du dernier prélèvement et le gain de poids quotidien moyen au cours de l'expérience de $+0,54$ pour le groupe de 43 animaux et de $+0,90$ pour le groupe des I 2 Charolais.

La possibilité d'utiliser la mesure du taux de racines de poils en anagène comme test d'aptitude à la croissance est discutée.

\section{INTRODUCTION}

Certaines caractéristiques biochimiques ou morphologiques des poils de bovins sont liées aux aptitudes zootechniques. BRochaR'T (I956) a montré que la surcharge calcique des poils était associée chez la Vache à une faible production laitière et à une baisse de fertilité ; cette surcharge calcique est fonction du temps, et liée à un 
faible taux de renouvellement du pelage. TURNER et SchlEGER (I960) avaient mis en évidence une relation négative entre l'épaisseur du pelage, mesurée selon une cotation subjective, et la croissance estivale de jeunes bovins à viande ; cette relation n'était pas due uniquement à la thermo-régulation, car elle persistait en partie après la tonte.

Ultérieurement (I970), ces mêmes auteurs ont montré que la composante du pelage en relation avec le gain de poids était la fréquence d'apparition des nouveaux poils, fréquence mesurée de façon objective, avec une technique élaborée, mais assez longue à mettre en ouvre ; ces auteurs ont obtenu une corrélation hautement significative de $+0,39$ (57 degrés de liberté) entre le gain de poids et la moyenne par individu de 9 déterminations effectuées au cours d'un an du nombre de nouveaux poils rapporté au nombre de follicules pileux par centimètre carré.

I1 nous a paru intéressant, à partir de ces deux séries d'observations de vérifier la relation établie par Turner et SCHLEGER, en utilisant une méthode plus simple, à savoir en déterminant directement la proportion de follicules pilctix en anagène, c'est-à-dire en phase d'activité, ce qui a été réalisé très simplement en examinant les racines de poils épilés.

Chez 1'Homme, cette analyse par trichogramme, maintenant couramment employée pour établir le diagnostic de la chute des cheveux dans les cas pathologiques, a même été proposée par BRADFIELI) et al. (I967) pour diagnostiquer le Kwashiarkor. Chez les animaux, la simplicité et l'exactitude de cette méthode ont conduit DONEY et SuITH (I969), chez le Mouton, à étudier l'action de divers facteurs causant l'arrêt de l'activité du follicule.

\section{MATÉRIEL E'T MÉTHODES}

Nous avons pu disposer de taurillons de quatre races faisant l'objet d'expériences sur l'efficacité de diverses rations d'engraissement ; quelques taureaux ayant présenté des troubles digestifs ou pathologiques chroniques ont été éliminés.

L'expérience a débuté en novembre I970. Les animaux ont été abattus, par lots constitués dès le début de l'expérience, à poids sensiblement égal de telle sorte que leur âge s'échelonnait entre I 5 et 18 mois à ce moment-là. Aussi l'abattage s'est-il étendu sur une assez longue période de mai à septembre I97I. Le gain de poicls vif, selon les groupes, a été compris entre I o48 et $1320 \mathrm{~g}$ par jour, la moyenne générale était de I $208 \mathrm{~g} / \mathrm{jour}$.

Quatre prélèvements de poils ont été effectués sur 55 taurillons, en décembre, mars, avril et le jour de l'abattage (mai à juillet), sauf pour un groupe de 12 taurillons charolais, pour lequel le dernier prélèvement a été réalisé le même jour (Io août) ; les taureaux de ce groupe ayant été abattus de o à 59 jours après ce prélèvement, ceci justifie le traitement statistique séparé qui a été appliqué au $4^{\mathrm{e}}$ prélèvement de ce dernier groupe.

L'épilation a été réalisée en arrière de l'épaule, au début de l'expérience par préhension des poils avec les doigts, puis, ultérieurement, à la pince porte-aiguille, méthode qui permet une épilation totale, notamment des poils courts, et a l'avantage d'éviter les artefacts dus à une épilation manuelle laborieuse (MAguire et KLIGMAN, 1964) ; l'opération dure trois minutes.

Les poils prélevés sont collés sur une bande de rouleau adhésif, la racine restant libre; les poils sont aisément stockés de cette façon. Pour l'examen, les racines (avec la partie adjacente de la tige du poil) sont coupées avec des ciseaux, dégraissées deux fois à l'alcool-éther sur un filtre métallique cylindrique à fond plat pourvu de trous très fins. Les racines peuvent alors être examinées directement à la loupe binoculaire (x 50 ) à sec, entre lame et lamelle, sans montage ni coloration. Toutefois, la différenciation entre les phases anagène et télogène (stade de repos du follicule pileux) est beaucoup plus facile après coloration de 30 minutes dans le carmin aluné (carmin I p. Ioo; alun de K 8 p. roo), qui colore les gaines épithéliales des racines en rouge violacé, 
alors que la racine kératinisée des poils en télogéne reste incolore, ou est très faiblement teintée (pl. I). Le pourcentage de racines de poils en anagène est calculé sur deux échantillons d'environ $4^{\text {oO }}$ racines chacun. L'ensemble de ces opérations nécessite environ une heure par échantillon.

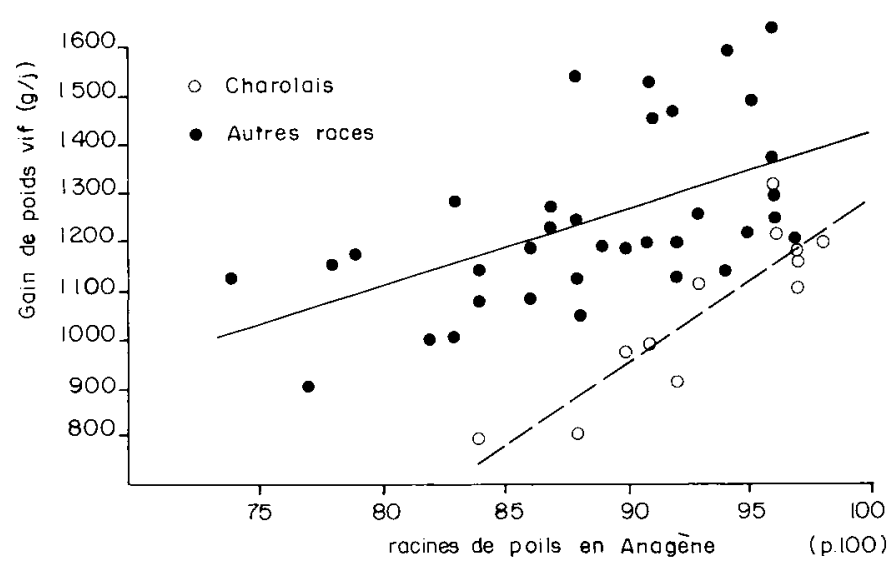

FIG. I. - Relation entre le taux de racines de poils en anagène et le gain de poids moyen au cours de l'expérience.

\section{RÉSULTATS}

Nous avons observé un taux très élevé de racines de poils en anagène, au cours des quatre prélèvements, avec toutefois une tendance à la baisse lors du dernier prélèvement (tabl. I).

TABLEAU I

Evolution du taux moyen de racines de poils en anagène au cours des quatre prélèvemonts successifs

\begin{tabular}{c|c|c|c|c}
\hline & Décembre & Mars & Avril & Mai-Août \\
\hline $\begin{array}{c}\text { Taux de racines de poils } \\
\text { en anagene }\end{array}$ & 95,9 & 94,4 & 97,2 & 89,0 \\
\hline
\end{tabular}

Nous n'avons pas observé de relation significative entre le taux de racines de poils en anagène des trois premiers prélèvements et le gain de poids quotidien moyen calculé à partir de la régression âge-poids durant toute l'expérience (respectivement $r=0,05 ; 0,27 ;-0,03)$. En revanche, des corrélations hautement significatives ont été mises en évidence pour le dernier prélèvement (tabl. 2 et fig. I).

La corrélation très élevée observée sur les I2 taureaux charolais peut être le fait du hasard; toutefois, certains facteurs ont pu contribuer à mieux extérioriser chez ce groupe la relation existant entre activité estivale des follicules pileux et croissance ; en effet : 
TABL,EAU 2

Corrélation entre le taux de racines de poils en anagène du dernier prélèvement de poils et le gain de poids

Le taux de racines de poils en anagène n'ayant pas une distribution normale, on a effectué une transformation arc sinus $\sqrt{\%}$

\begin{tabular}{|c|c|c|c|c|}
\hline $\begin{array}{l}\text { Moment du dernier } \\
\text { prólevement de poils }\end{array}$ & $\begin{array}{c}\text { Nombre } \\
\text { d'animaux }\end{array}$ & Race & \begin{tabular}{|c|} 
Régimes \\
alimentaires
\end{tabular} & $\begin{array}{l}\text { Corrélation entre taux de racines } \\
\text { de poils en anagène du dernier } \\
\text { prélèvement et gain de poids } \\
\text { quotidien moyen au cours de } \\
\text { l'expérience }\end{array}$ \\
\hline $\begin{array}{l}\text { Lors de l'abattage } \\
\text { (mai à juillet) }\end{array}$ & 43 & $\begin{array}{l}22 \text { Croisés Charolais } \\
12 \text { Montbéliards } \\
9 \text { Salers }\end{array}$ & $\begin{array}{l}7 \text { régimes } \\
\text { différents }\end{array}$ & $(p<0,01)$ \\
\hline $\begin{array}{c}10 \text { août } \\
\text { (0 à } 59 \text { jours avant } \\
\text { l'abattage) }\end{array}$ & 12 & Charolais & unique & $\begin{array}{c}0,90 \\
(p<0,01)\end{array}$ \\
\hline
\end{tabular}

$I^{\circ}$ les prélèvements de poils ont été effectués pour tous les animaux le même jour du mois d'août, en pleine période estivale, pendant laquelle, selon nos résultats, le taux de follicules en activité a tendance à baisser ;

$2^{\circ}$ ce groupe était homogène du point de vue racial ;

$3^{\circ}$ les animaux de ce groupe avaient tous le même régime alimentaire.

\section{DISCUSSION}

L'absence de relation significative entre le taux de racines de poils en anagène et le gain de poids pour les trois premiers prélèvements effectués en hiver et au début du printemps est peut-être due au fait qu'à cette saison le taux de racines en anagène est très élevé, et masque d'éventuelles différences individuelles, qui apparaissent, en revanche, lorsque l'activité des follicules pileux se ralentit. De plus, en été, une épaisseur excessive du pelage, liée à un ralentissement de l'activité des follicules, peut devenir un facteur limitant de la thermo-régulation et par suite de la croissance, comme l'ont montré TURNER et SCHLEGER. Toutefois, ces auteurs ont également montré que le pelage et la croissance sont liés par d'autres facteurs que la seule possibilité de thermo-régulation, et formulé l'hypothèse que la croissance corporelle et l'activité des follicules pileux seraient contrôlées par un mécanisme commun, qui pourrait être endocrinien. Le taux de racines de poils en anagène du $4^{\mathrm{e}}$ prélèvement est en corrélation positive avec la croissance quotidienne moyenne observée sur la totalité de la période expérimentale ( 7 à II mois) ; en revanche, il n'est pas en corrélation avec la croissance observée pendant les $5_{5}$ à 30 jours précédant l'abattage ; ceci semble indiquer que le taux de racines en anagène du $4^{\text {e }}$ prélèvement n'est pas le reflet 
d'une période limitée, mais l'expression d'une aptitude intrinsèque à la croissance. Dans cette hypothèse, la mesure du taux de racines de poils en anagène pourrait peut-être servir de test pratique d'aptitude à la croissance, dans la mesure où ce taux serait mesuré sur les jeunes veaux en été, avant la période hivernale de croissance active du pelage; la vérification de cette hypothèse est en cours.

$$
\text { Reçu pour publication en septembre } 1972 .
$$

\title{
REMERCIEMENTS
}

Nous tenons à remercier la Station de l'Élevage des Ruminants du C. R. Z. V. de Theix, pour les facilités expérimentales et les données mises à notre disposition.

\author{
SUMMARY
}

\author{
RELATIONSHIP BETWEEN HAIR FOLLICLE ACTIVITY \\ AND BODY WEIGHT GAIN IN BEEF CATTLE
}

The experiment was carried out with 55 young bulls of 4 different breeds subjected to various diets. The hair follicle activity was measured indirectly by means of the percentage of hair roots in anagen growth phase obtained by plucking. Four series of hair samplings were made during the fattening period in December, March and April. The last one took place, on the one hand at slaughter, $i$. $e$. between May and July for 43 animals of various breeds and diets, and, on the other hand $o$ to 59 days before slaughter, on August Ioth, for I2 Charolais receiving the same diet.

As regards the first three series of samples, the percentage of hair roots in anagen phase was very high, whereas a reduction was observed for the last sampling. Highly significant correlations were obtained between the percentage of hair roots in anagen phase at the last sampling and the mean weight gain during the experiment, $i . e .+0.54$ for the group of 43 animals and +0.90 for the group of 12 Charolais.

The possibility of using the percentage of hair roots in anagen phase as a growth capacity test is discussed.

\section{RÉFÉRENCES BIBLIOGRAPHIQUES}

Bradfield R. B., Bailly M. A., Margen S., I967. Morphological changes in human scalp hair roots during deprivation of protein. Science, 15\%, 438-439.

Brochart M., r957. L'exploration de la nutrition phospho-calcique des vaches laitières au moyen du dosage de Ca et $\mathrm{P}$ dans les poils. Ann. Zootech., 6, $\mathrm{I}_{5} \mathrm{I}-235$.

Doney J.-M., Smith W. F., I969. Casting of the fleece in sheep. Estimation of experimentally induced fibre shedding rate. J. agric. Sci. Camb., $73,23 \mathrm{I}-237$.

Maguire H. C., Kligman A. M., I964. Hair plucking as a diagnostic tool. J. Invest. Derm., 43, 77-79.

Turner H. G., Schleger A. V., I960. The significance of coat type in cattle. Austr. J. agric. Res., 11, $645-663$.

Turner H. G., Schleger A. V., I97o. An analysis of growth process in cattle coats and their relation; to coat type and body weight gain. Austr. J. biol. Sci., 23, 201-218. 


\section{PLANCHE I}

Différenciation des stades anagène et télogène par le carmin aluné.

Les racines de poils en anagène sont colorées tandis que celles au stade télogène $(\rightarrow)$ sont restées incolores. 


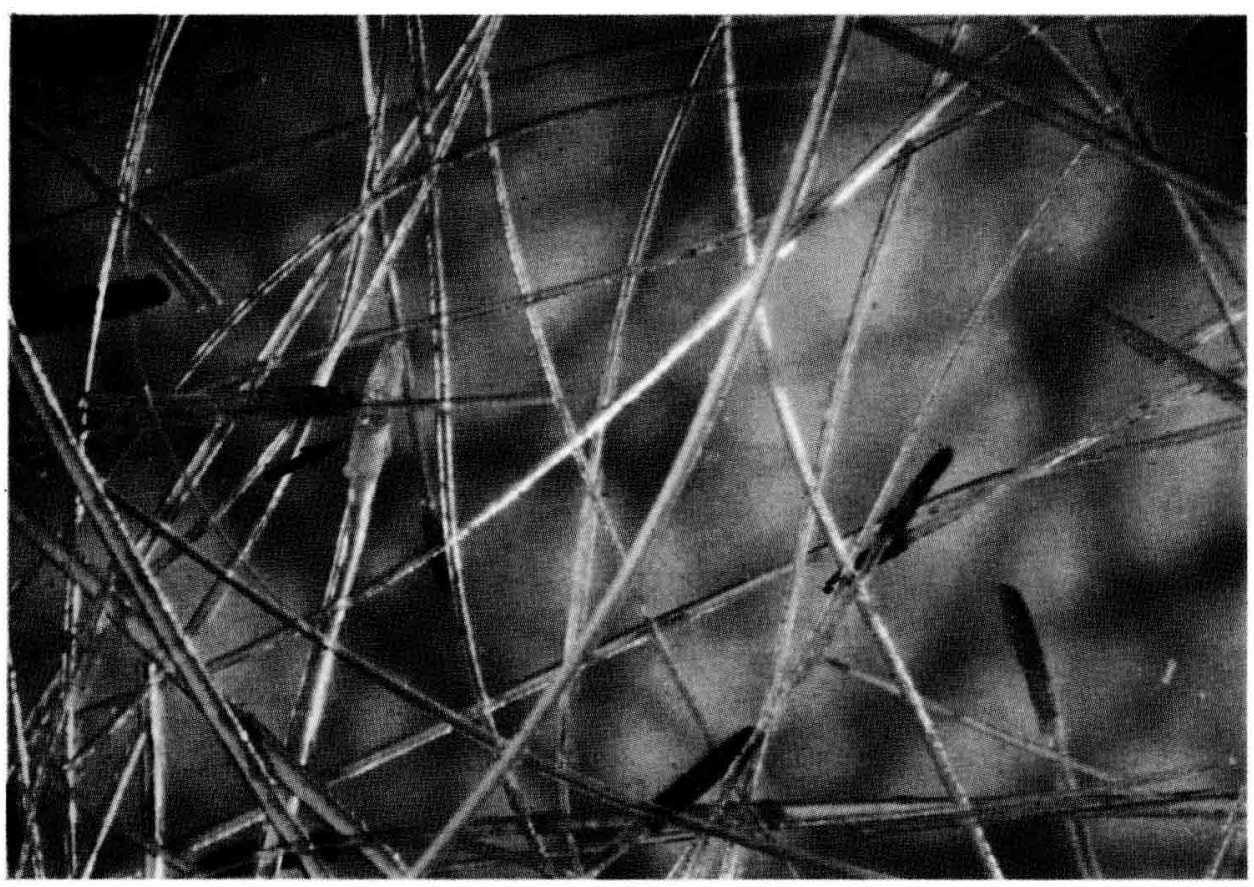

